\title{
A characteristic initial value problem for the Euler-Poisson-Darboux equation.
}

\author{
Eutiquio O. Young (Florida U.S.A.) (*) (**)
}

Summary. - An explit solution is obtained for a charasteristic initial value problem for the Euler-Poisson-Darboux equation by Riesz method of analytic continuation of a generalized Riemann.Liouville integral.

\section{1. - Introduction.}

This paper is concerned with an interior characteristic initial value problem for the nonhomogeneous EULER-PoIsson-DarBodx equation

$$
L_{x} u \equiv \frac{\partial^{2} u}{\partial x_{1}^{2}}+\frac{k}{x_{1}} \frac{\partial u}{\partial x_{1}}-\sum_{i=1}^{m} \frac{\partial^{2} u}{\partial x_{i}^{2}}=f\left(x_{1}, \ldots, x_{m}\right)
$$

in which the solution $u$ is required to satisfy a vanishing initial condition

$$
u\left(x_{1}, \ldots, x_{m}\right)=0
$$

on the surface of the initial characteristic cone

$$
S: x_{1}^{2}-x_{2}^{2}-\ldots-x_{m}^{2}=0, \quad x_{1} \geq 0 .
$$

The parameter $k$ is assumed real and satisfies the condition $k>m-2$ if $m$ is even and $k>m-3$ if $m$ is odd. This restriction on $k$ is made in order that the integral solution may converge. However, the uniqueness of the solution of the problem (1), (2) can be proved for $k \geq 0$ [1, pp. 645.47].

When $k=0$, equation (1) reduces to the wave equation for which the solution of the present prohlem was given by Riesz [2, pp. 107-125] (see also [3]). In the cases $m=2$ and $m=3$ for which $k>0$, it will be evident that by letting $k$ approach zero, the solution of (1), (2) reduces to the solution of the corresponding problem for the wave equation. However, in view of the restriction on $k$, it is not at all evident that for general $m$ one can arrive at the problem for the wave equation by such a limiting process.

(*) This work was supported by NSF research grant GP-11543.

(**) Entrata in Redazione il 18 ottobre 1969. 
The procedure used here is similar to that employed in [4] and [5] which is based on analytic continuation of a generalized RIEMANN-LIOUviLLe inte. gral developed by Rresz [2].

Let $x$ and $y$ denote two points in $m$-dimensional space-time or LORENTzspace with coordinates $x_{I}, \ldots, x_{m}$ and $y_{1}, \ldots, y_{m}$, respectively. The square of the (LoREnTz) distance between these points is given by

$$
R=\left(y_{1}-x_{1}\right)^{2}-\left(y_{2}-x_{2}\right)^{2}-\ldots-\left(y_{m}-x_{m}\right)^{2} .
$$

If $x$ is fixed and $y$ a variable point, then the equation $R=0$ with $x_{1}-y_{1}>0$ defines the retrogade characteristic cone of equation (1) with vertex $x$. On the other hand, $R=0$ with $x_{1}-y_{1}<0$ defines the direct or upright charac. teristic cone with the same vertex.

In the sequel, we shall also ase $x$ to denote the position vector of the point $\left(x_{1}, \ldots, x_{m}\right)$. Let $\beta$ and $\gamma_{n}$ be two vectors with respective components $\beta_{1}, \ldots, \beta_{m}$ and $\gamma_{1}, \ldots, \gamma_{m}$. The scalar product $(\beta, \gamma)$ of $\beta$ and $\gamma$ is defined by

$$
(\beta, \gamma)=\beta_{1} \gamma_{1}-\beta_{2} \gamma_{2}-\ldots-\beta_{m} \gamma_{m} \text {. }
$$

In analogy with ordinary Euclidean space, the vectors $\beta$ and $\gamma$ are said to be orthogonal if $(\beta, \gamma)=0$. A vector $\beta$ is said to be timelike if $(\beta, \beta)>0$ and spacelike if $(\beta, \beta)<0$. In particular, a vector $\beta$ is called a timelike univ vector or a spacelike unit vector according as $(\beta, \beta)=1$ or $(\beta, \beta)=-1$.

\section{2. - The Solntion Formula.}

Let $x$ be a point in the interior of the direct initial cone (3) and let $C^{x}$ denote the retrogade characteristic cone with vertex $x$, that is,

$$
C^{x}: R=\left(y_{1}-x_{1}\right)^{2}-\ldots-\left(y_{m}-x_{m}\right)^{2}=0, \quad y_{m}-x_{m}<0 .
$$

Denote by $D$ the domain enclosed by $C^{x}$ and the initial cone $S$. The line joining the vertices of $C^{x}$ and $S$ will be called the axis of $D$. If $u$ and $v$ are two functions with continuous second derivatives in $D$, then by Grees's theorem,

$$
\int_{D}\left[u M_{y} v-v L_{y} u\right] d y=\int_{S+C^{x}}\left(u \frac{\partial v}{\partial n}-v \frac{\partial u}{\partial n}-\frac{k u v}{y_{1}}\right) d S
$$

where $d y=d y_{1} \ldots d y_{m}$ denotes the volume element in $D$ and $d S$ denotes the surface element on $S$ and $C^{x}$. The operator

$$
M_{y}=\frac{\partial^{2}}{\partial y_{1}^{2}}-\frac{k}{y_{1}} \frac{\partial}{\partial y_{1}}+\frac{k}{y_{1}^{2}}-\sum_{i=2}^{m} \frac{\partial^{2}}{\partial y_{i}^{2}}
$$

is the adjoint of $L$. 
We now set $v=V^{\alpha+2}(x ; y)$ in $(5)$ where

$$
V^{\alpha+2}(x ; y)=\frac{2^{k} y_{1}^{k} R^{(\alpha+2-m))^{2}}}{H_{m}(\alpha+2) R^{\prime k l^{2}}} F\left(\frac{k}{2}, \frac{\alpha+2+k-m}{2} ; \frac{\alpha+4-m}{2} ; \frac{R}{R^{\prime}}\right)
$$

with

$$
R^{\prime}=\left(y_{1}+x_{1}\right)^{2}-\left(y_{2}-x_{2}\right)^{2}-\ldots-\left(y_{m}-x_{m}\right)^{2}=R+4 x_{1} y_{1}
$$

and

$$
H_{m}(\alpha)=2^{\alpha-1} \pi^{(m-2) / 2} \Gamma\left(\frac{\alpha}{2}\right) \Gamma\left(\frac{\alpha+2-m}{2}\right)
$$

The funetion (6) was determined in [4] and also in [6]. For $\alpha$ sufficient large, $V^{x+2}$ satisfies the conditions

(7) (i) $L_{x} V^{\alpha+2}(x ; y)=V^{\alpha}(x ; y)$

(ii) $M_{y} V^{\alpha+2}(x ; y)=V^{\alpha}(x ; y)$

and vanishes togeter with its derivatives on the surface of $C^{x}$. Writing

$$
I^{x} u(x)=\int_{D} u(y) V^{x}(x ; y d y
$$

and using (7ii) together with (1) and (2), we obtain from (5) the formula

$$
I^{\alpha} u(x)=\int_{D} f(y)^{\alpha+2}(x ; y) d y
$$

It is clear that the integral (9) converges for $\alpha>m$ and $k \geq 0$. Under this condition it follows from (7i) that

$$
L_{x}\left[\boldsymbol{l}^{x} u(x)\right]=\int_{D} f(y) L_{x} V^{\alpha+2}(x ; y) d y=\int_{D} f(y) V^{\alpha}(x ; y) d y=I^{\alpha} f(x)
$$

As will be shown in the next section, the integral (8) ean be continued analytically with respect to $\alpha$ for $k>m-2$ and gives

$$
I^{0} u(x)=u(x)
$$

verifying equation (1). Further, the analytic continuation of (9) to $\alpha=0$ will yield the explicit solution

$$
u(x)=I^{0+2} /(y)
$$


This will be shown to vanish as $x$ approaches the surface of the initial cone (3), thas verifying (2). It is in this sense that formula (9) provides the solution of the problem (1), (2).

\section{3. - The Analytic Continuation of $I^{x} u(x)$.}

In order to be able to perform the analytic continuation of the integral (8) with respect to $\alpha$, we introduce a suitable coordinate system in $D$ and assume that $u$ is at least $[m / 2]$ times continuously differentiable. We adapt here a coordinate system that was devised by Riesz [7]. Let a be a fixed timelike unit vector issuing from $x$ and pointing toward the origin, and let $\beta$ be a rariable spacelike unit vector orthogonal to a. The vector $\beta$ actually lies in an $(m-1)$-dimensional plane which passes through $x$ and is parallel to the plane where the cones $C^{x}$ and $S$ meet. Thus we have

$$
(\alpha, a)=1, \quad(\beta, \beta)=-1, \quad(a, \beta)=0
$$

If the vector $\beta$ issues from the origin, its endpoint describes a «unit sphere» $\omega_{m-1}$ defined by $\beta_{1}^{2}-\beta_{2}^{2}-\ldots-\beta_{m}^{2}=-1$ and lying in an $(m-1)$-plane ortho. gonal to the vector $x$. Let $y$ be the position vector of an arbitrary point $y$ in $D$. Then we can write

$$
y-x=t a+\rho \beta
$$

where $t$ and $\rho$ are nonnegative real numbers. This expression can be put in the form

$$
y-x=r(\gamma+s \tau)
$$

where $\gamma$ and $\tau$ are vectors defined by

$$
\gamma=\frac{1}{2}(\alpha+\beta), \quad \tau=\frac{1}{2}(a-\beta)
$$

and $r$ and $s$ are scalars related to $t$ and $\rho$ by the equations

$$
r=t+\rho, \quad s=\frac{t-\rho}{t+\rho} .
$$

The inverse formulas of (17) are given by

$$
t=\frac{1}{2} r(1+s), \quad p=\frac{1}{2} r(1-s) .
$$


The variables $r, s$ and the angular variable $\beta$ will now be used as the new coordinates in $D$. In this coordinate system, the square of the LoRENTz distance (4) becomes

$$
R=(y-x, y-x)=t^{2}-\rho^{2}=(t+\rho)^{2} \frac{t-\rho}{t+\rho}=r^{2} s
$$

by virtue of (14) and (15). When $y$ lies on the cone $C^{x}, R=r^{2} s=0$. Since the vertex $x$ is given by the single equation $r=0$, it follows that the cone $C^{x}$ apart from the vertex is given by $s=0$. Points on the axis $y=x+t a$ (or $p=0$ ) correspond to $s=1$. Moreover, for $r=c=$ constant, the equation $t+\rho=r=c$ defines a direct characteristic cone with vertex $y=x+c a$. Thus the initial cone $S$ is defined by the equation $r=r_{0}$, where $r_{0}=$ $=\left(x_{1}^{2}-x_{2}^{2}-\ldots-x_{m}^{2}\right)^{z / 2}$ is distance of $x$ from the origin. Hence the domain $D$ including its boundary is characterized by the inequalities

$$
0 \leq s \leq 1, \quad 0 \leq r \leq r_{0} .
$$

If we denote as usual the surface element on the "unit sphere» $\omega_{m-1}$ by $d \omega_{m-1}$, then the element of volume in $D$ is given by

$$
d y=\rho^{m-2} d \rho d l d \omega_{m-1}
$$

On changing from the variables $\rho, t$ to the variables $r, s$ using (18), this becomes

$$
d y=2^{1-m} r^{m-1}(1-s)^{m-2} d r d s d \omega_{m-1} .
$$

We now proceed to prove (1.1). We distinguish between the cases $m$ even and $m$ odd, concerning ourselves here only with the case $m$ even, $m \geq 2$. We expand in infinite series the hypergeometric function appearingin $V^{a}$ of the integral (8) and consider the general term of the first $\frac{m}{2}$ terms, namely

$$
I_{j}^{\alpha} u(x)=\frac{C_{j}(\alpha)}{\Gamma\left(\frac{\alpha+2-m}{2}+j\right)} \int_{\dot{D}}^{\tau} \frac{y_{1}^{k} u(y) R^{(\alpha-m+2 j) / 2}}{R^{i k / 2+j}} d y
$$

$0 \leq j \leq \frac{m-2}{2}$, where

$$
C_{j}(\alpha)=\frac{\Gamma\left(\frac{\alpha+2-m}{2}\right) \Gamma\left(\frac{k}{2}+j\right) \Gamma\left(\frac{\alpha+k-m}{2}+j\right) 2^{k}}{\Gamma\left(\frac{k}{2}\right) \Gamma\left(\frac{\alpha+k-m}{2}\right) H_{m}(\alpha) j !} .
$$


The leading term corresponding to $j=0$ is given by

$$
\begin{aligned}
I_{0}^{\alpha} u(x) & =\frac{2^{k}}{H_{m}(\alpha)} \int_{D} \frac{y_{1}^{k} u(y) R^{(x-m) / 2}}{R^{\prime k / 2}} d y \\
& =\frac{1}{H_{m}(\alpha)} \int_{D} g(y) R^{(\alpha-m) / 2} \\
& =J^{x} g(x)
\end{aligned}
$$

where we have set

$$
g(y)=\frac{2^{k} y_{1}^{k} u(y)}{R^{k / 2}}
$$

The last integral $J^{x} g^{\prime}(x)$, which converges for $\alpha>m-2$ and $k>m-2$, is identical with that considered in [2, p. 111] for which it is true that $\alpha$ can be continued analytically to $\alpha=0$, giving the result

$$
J^{0} g(x)=g(x)
$$

Hence,

$$
I_{0}^{0} u(x)=g(x)=\frac{2^{k} x_{1}^{k} u(x)}{\left[\left(2 x_{1}\right)^{2}\right]^{k / 2}}=u(x)
$$

It remains to be shown that all the remaining terms in the expansion of $I^{\alpha} u(x)$ vanish as $\alpha$ is continued analytically to $\alpha=0$.

For $1 \leq j \leq \frac{m-2}{2}$, wo write $(21)$ in the now coordinates $r, s$, and $\beta$ using (16), (19), and (20), obtaining

$$
I_{j}^{\alpha} u(x)=\frac{C_{j}(\alpha) 2^{1-m}}{\Gamma\left(\frac{\alpha+2-m}{2}+j\right)} \int_{0^{\circ}}^{r_{0}} r^{\alpha+2 j-1} d r \int_{0}^{1} M(h)(1-s)^{m-2} s^{(\alpha-m+2 j) / 2} d s .
$$

Here

$$
M(h)=\int_{\omega_{m-1}} h(r, s) d \omega_{m-1}
$$

with

$$
\mathrm{h}(r, s)=\frac{\left[x_{1}+r\left(\gamma_{1}+s \tau_{1}\right)\right]^{k} u[x+r(\gamma+s \tau)]}{\left\{r^{2} s+4 x_{1}\left[x_{1}+r\left(\gamma_{1}+s \tau_{1}\right)\right]\right\}^{k / 2}}
$$

With the differentiability condition required of $u$ aud with $k>m-2$, inte- 
gration by parts with respeci to $s$ in the inner integral of (22) can be performed $\frac{m-2 j}{2}$ times, yielding the result

$$
\begin{aligned}
I^{\alpha} u(x) & =\frac{C_{j}(\alpha) 2^{1-m}}{\Gamma\left(\frac{\alpha+2}{2}\right)}(-1)^{\left.(m-2 j)\right|^{2}} \int_{0}^{r_{0}} r^{\alpha+2 j-1} d r \\
& \times \int_{0}^{1} s^{\alpha / 2}\left(\frac{\partial}{\partial s}\right)^{(m-2 j) / 2}\left[M(h)(1-s)^{m-2}\right] d s
\end{aligned}
$$

The integral (24) is now convergent for $\alpha>-2$ and $k>m-2$. Hence letting $\alpha \rightarrow 0$, it follows that $I_{j}^{0} u(x)=0,1 \leq j \leq \frac{m-2}{2}$, since $C_{j}(0)=0$ in wiew of the factor $\Gamma(\alpha / 2)$ in $H_{m}(\alpha)$.

The remaining terms in the series expansion of the bypergeometric function can be expressed as

$$
A\left(\frac{R}{R^{\prime}}\right)^{m / 2}{ }_{3} F_{2}\left(\frac{R}{R^{\prime}}\right)
$$

where $A$ is some constant and ${ }_{3} F_{2}$ is a generalized hypergeometric funotion. Substitating this for the hypergeometric function in (8), we see that the resulting integral

$$
I_{*}^{\alpha} u(x)=\frac{2^{k} A}{H_{m}(\alpha)} \int_{D} \frac{y_{1}^{k} u(y) R^{k / 2}}{R^{\prime(k+m) / 2}}{ }_{3} F_{2}\left(\frac{R}{R^{\prime}}\right) d y
$$

converges for $\alpha>-2$ and $k>m-2$. For the same reason as above, it fol. lows that this integral vanishes as $\alpha$ is allowed to approach zero. This establishes equation (11).

In view of what has just been proved, we can now conclude from (10) and (12) that (9) indeed satisfies equation (1).

\section{4. - The Explicit Solution.}

We now perform the analitic continuation with respect to $\alpha$ in the integral (9) to arrive at a explicit solution of the problem (1), (2). For this purpose we require that the function $f$ has continuous derivatives up to the order $\left[\frac{m+2}{2}\right]$. In the cases $m=2$ and $m=3$, we readily obtain the solution of 
(1), (2) by simply setting $\alpha=0$ in (9). In fact, when $m=2$ we have

$$
u(t, x)=2^{k-1} \int_{D} \frac{\tau^{k} f(\tau, \xi)}{R^{\prime k^{2}}} F\left(\frac{k}{2}, \frac{k}{2} ; 1 ; \frac{R}{R^{\prime}}\right) d \xi d \tau
$$

where $R=(\tau-t)^{2}-(\xi-x)^{2}, R^{\prime}=(\tau+t)^{2}-(\xi-x)^{2}$, and $D$ is the rectangle bounded by the characteristic lines $\xi=\tau, \xi=-\tau, \xi+\tau=x+t$, and $\xi-\tau=$ $=x-t$. The solution (25) is valid for $k \geq 0$ and yields the solution of the corresponding problem for the wave equation $u_{t t}-u_{x x}=0$ where $k=0$. In characteristic coordinates $s=\xi+\tau, r=\xi-\tau$ with $s_{0}=x+t, r_{0}=x-t$, (25) assumes a more familiar form (see [8])

(26) $u\left(s_{0}, r_{0}\right)=\frac{1}{4} \int_{0}^{s_{0}} \int_{0}^{r_{0}} \frac{(s-r)^{k}}{\left[\left(r-s_{0}\right)\left(r_{0}-s\right)\right]^{k / 2}} f\left(\frac{s+r}{2}, \frac{s-r}{2}\right) \times F\left(\frac{k}{2}, \frac{k}{2} ; 1 ; z\right) d r d s$

where $z=\left(r-r_{0}\right)\left(s-s_{0}\right) /\left[\left(r-s_{0}\right)\left(s-r_{0}\right)\right]$.

It is easily seen from (26) that $u$ vanishes on the characteristic lines $s=0$ and $r=0$.

When $m=3$, we obtain from $(9)$

$$
u(t, x, y)=\frac{2^{k-1}}{\pi} \int_{D} \frac{\tau^{k} f(\tau, \xi, \eta)}{R^{1 / 2} R^{\prime k / 2}} F\left(\frac{k-1}{2}, \frac{k}{2} ; \frac{1}{2} ; \frac{R}{R^{\prime}}\right) d \xi d r_{1} d \tau
$$

where $R=(\tau-t)^{2}-(\xi-y)^{2}-(\eta-y)^{2}, R^{\prime}=(\tau+t)^{2}-(\xi-x)^{2}-(\eta-y)^{2}$ and $D$ is the domain bounded by the retrogade characteristic cone $R=0, t-\tau>0$ and the direct characteristic cone $\tau^{2}-\xi^{2}-\eta^{2}=0, \tau>0$. This solution is $k \geq 0$.

Thus we need continue (9) only in the case $m \geq 4$. We consider here the case when $m$ is even and simply state the result for $m$ odd, $m \geq 5$. Expanding the hypergeometric function appearing in $V^{x+2}$ on the right side of (9), we valid for consider the general term of the first $(m-4) / 2$ terms,

$$
U_{j}(\alpha)=\frac{B_{j}(\alpha)}{\Gamma\left(\frac{\alpha+4-m}{2}+j\right)} \int \frac{y_{j}^{k} f(y) R^{(x+2-m+2 j) / 2}}{R^{\prime k \mid 2+j}} d y
$$

$0 \leq j \leq \frac{m-4}{2}$, where

$$
B_{j}(\alpha)=\frac{2^{k} \Gamma\left(\frac{\alpha+4-m}{2}\right) \Gamma\left(\frac{k}{2}+j\right) \Gamma\left(\frac{\alpha+k+2-m}{2}+j\right)}{H_{m}(\alpha+2) \Gamma\left(\frac{k}{2}\right) \Gamma\left(\frac{\alpha+k+2-m}{2}\right) j !}
$$


In the new coordinates $r, s$, and $\beta,(28)$ becomes

$$
U_{j}(\alpha)=\frac{2^{1-m} B_{j}(\alpha)}{\Gamma\left(\frac{\alpha+4-m}{2}+j\right)_{0}} \int_{0}^{r_{0}} r^{\alpha+2 j+1} d r \int_{0}^{l} M(h)(1-s)^{m-2} s^{(\alpha+2-m+2 j) / 2} d s
$$

where $M$ is as defined in (23) with $f$ replacing $u$ in the expression for $h$. Under the differentiability condition imposed on $f$, we can perform integra. tion by parts with respect to $s \frac{m-2-2 j}{2}$ times. The resulting integral (see equation (24)) converges for $\alpha>-2$ and $k>m-2$. Thus letting $\alpha \rightarrow 0$, we obtain the result

$$
U_{j}(0)=(-1)^{(m-2 j) / 2} 2^{1-m} B_{j}(0) \int_{0}^{T_{0}} r^{2 j+1} \times\left\{\left(\frac{\partial}{\partial s}\right)^{(m-4-2 j) / 2}\left[M(h)(1-s)^{m-2}\right]\right\}_{s=0} d r
$$

with

$$
B_{j}(0)=\frac{2^{k-1} \Gamma\left(\frac{k}{2}+j\right) \Gamma\left(\frac{k+2-m}{2}+j\right)}{\Pi^{(m-2) / 2} \Gamma\left(\frac{k}{2}\right) \Gamma\left(\frac{k+2-m}{2}\right) j !}, \quad 0 \leq j \leq \frac{m-4}{2} .
$$

Let $b=\frac{k}{2}, c=\frac{k+\alpha+2-m}{2}, d=\frac{\alpha+4-m}{2}, v=\frac{m-2}{2}$ and put $z=$ $\frac{R}{K^{\prime}}$. The remaining term in the expansion of the of the hypergeometric function of $V^{\alpha+2}$ can be expressed as

$$
\begin{aligned}
F^{*}(z) & =\frac{(b)_{\nu}(c)_{\nu}}{(d)_{\nu}} \frac{z^{\nu}}{v !}\left[1+\frac{(b+v)(c+v)}{(d+v)(\nu+1)} z+\ldots\right] \\
& =\frac{(b)_{\nu}(c)_{\nu}}{(d)_{\nu}} \frac{z^{\nu}}{v !}{ }_{3} F_{2}(b+\nu, c+\nu, 1 ; d+\nu ; \nu+1 ; z)
\end{aligned}
$$

where $(\lambda)_{\nu}=\Gamma(\lambda+v) / \Gamma(\lambda)$. The generalized hypergeometric function ${ }_{3} F_{2}(z)$ con. verges uniformly in $z$ for $z \leq \sigma$ with any $\sigma<1$. Hence for the remaining terms in the expansion of (9) we have

$$
W(\alpha)=A(\alpha) \int \frac{y_{1}^{k} f(y) R^{\alpha / 2}}{R^{\prime(k+m-2 / 2)}}{ }^{3} F_{2}\left(\frac{k+m-2}{2}, \frac{\alpha+k}{2}, 1 ; \frac{\alpha+2}{2} ; \frac{m}{2} ; \frac{R}{R^{\prime}}\right) d y
$$

with $A$ denoting all constant factors. This integral converges uniformly for 
$\alpha>-2$ and $k>m-2$. So letting $\alpha \rightarrow 0$, we have

$$
W(0)=A(0) \int \frac{y_{1}^{k} f(y)}{R^{(k+m-2) / 2}} F\left(\frac{k}{2}, \frac{k+m-2}{2} ; \frac{m}{2} ; \frac{R}{R^{\prime}}\right) d y
$$

with

$$
A(0)=\frac{2^{k-1} \Gamma\left(\frac{k+m-2}{2}\right)}{\Pi^{(m-2) / 2} \Gamma\left(\frac{k+2-m}{2}\right) \Gamma\left(\frac{m}{2}\right)} .
$$

Therefore for $m$ even, $m \geq 4$, and $k>m-2$, the solution of the problem (1), (2) is

$$
\begin{aligned}
u(x) & =\frac{2^{k-m}}{\Pi^{(m-2) / 2}} \sum_{j=0}^{(m-4) / 2}(-1)^{(m-2 j) / 2} \frac{\Gamma\left(\frac{k}{2}+j\right) \Gamma\left(\frac{k+2-m}{2}+j\right)}{\Gamma\left(\frac{k}{2}\right) \Gamma\left(\frac{k+2-m}{2}\right) j !} \\
& \times \int_{0}^{r_{0}} r^{2 j+1}\left\{\left(\frac{\partial}{\partial s}\right)^{(m-4-2 j) / 2}\left[M(h)(1-\tau)^{m-2}\right]\right\}_{s=0} d r \\
& +\frac{2^{k-1} \Gamma\left(\frac{k+m-2}{2}\right)}{\Pi^{(m-2) / 2} \Gamma\left(\frac{k+\frac{k+m}{2}-m}{2}\right) \Gamma\left(\frac{m}{2}\right)} \int_{D} \frac{y_{1}^{k} f(y)}{R^{(k+m-2) / 2}} F\left(\frac{k}{2} \cdot \frac{k+m-2}{2} ; \frac{m}{2} ; \frac{R}{R^{\prime}}\right) d y .
\end{aligned}
$$

The case $m$ odd, $m \geq 5$, and $k>m-3$ can be treated in the same way. We obtain the result

$$
\begin{aligned}
u(x) & =\frac{2^{k-m}}{\Pi^{(m-1) / 2}} \sum_{j=0}^{(m-5) / 2}\left(-1 j^{(m-3-j) / 2} \frac{\Gamma\left(\frac{k}{2}+j\right) \Gamma\left(\frac{k+2-m}{2}+j\right)}{\Gamma\left(\frac{k}{2}\right) \Gamma\left(\frac{k+2-m}{2}\right) j !}\right. \\
& \times \int_{0}^{r_{0}} r^{2 j+1} \int_{0}^{l} s^{-1 / 2}\left(\frac{\partial}{2 s}\right)^{(m-3-2 j) / 2}\left[M(h)(1-s)^{m-2}\right] d s d r \\
& +\frac{2^{k-1} \Gamma\left(\frac{k-1}{2}\right) \Gamma\left(\frac{m-3+k}{2}\right)}{\Pi^{(m-1) / 2} \Gamma\left(\frac{k}{2}\right) \Gamma\left(\frac{2-m+k}{2} \Gamma\left(\frac{m-1}{2}\right)\right.} \int_{j} \frac{y_{1}^{k} f(y) R^{-1 / 2}}{R^{(m+k-3) / 2}} \\
& \times{ }_{3} F_{2}\left(\frac{k-1}{2}, \frac{k+m-3}{2} ; 1 ; \frac{1}{2}, \frac{m-1}{2} ; \frac{R}{R^{\prime}}\right) d y .
\end{aligned}
$$


That the solution (27) for $m=3$ or (30) for $m$ even, $m \geq 4$, or (31) for $m$ odd, $m \geq 5$, satisfies the initial condition (2) follows readily in the new coordinates $r, s, \beta$ from the observation that as $x$ approaches the initial cone $(3), r_{0}$ tends to zero and vice versa.

\section{REFERENCES}

[1] $\mathrm{R}$ Courant and D. Hicbert, Methods of Mathematical Physics, Interscience Publishers, New York, 1962.

[2] M. Riesz, L'integrale de Riemann Lionville et le problem de Oauchy, Acta Math., 81 (1948), 1.223.

[3] E. C. Young, The charactsristic initial value problem for the vawe equation in $n$ dimensions, J. Math. \& Mech, 17 (1968), 885-890.

[4] R. DAvis, On a regular Canchy problem for the Euler-Poisson-Darboux equation, Annali di Mat, 42 (1956). 205.226.

[5] E. C. Young, A solution of the singular Cauchy problem for the non-homogeneous FulerPoisson-Darboux equation, J. Diff. Eqs., 3 (1967), 522545.

[6] - - On a method of determining the Riesz kernel for the Euler-Poisson-Darboux operator, J. Math. Anal. \& App, 16 (1966), 35̃ó362.

[7] M. RiEsz, The aualytic continuation of the Rismann Liouville integral in the hyperbolic case, Canadian J. Math., 13 (1961), 37.47.

[8] P. GERMAIN and R. BADER, Sur quelques problèmes aux iimites, singuliers pour une équation hyperbolique, Compt. Rend. Acad. Sei., Paris, 231 (1950), 268.270. 